\title{
Analysis of risk factors and clinical sequelae of direct electrical cortical stimulation-induced seizures and afterdischarges in patients undergoing awake mapping
}

\author{
Zachary A. Abecassis, MD, ${ }^{1}$ Amit B. Ayer, MD, MBA, ${ }^{2}$ Jessica W. Templer, MD, ${ }^{3}$ \\ Ketan Yerneni, BA, ${ }^{1}$ Nikhil K. Murthy, MD, MS, ${ }^{2}$ and Matthew C. Tate, MD, PhD ${ }^{2,3}$ \\ ${ }^{1}$ Feinberg School of Medicine, Northwestern University; and Departments of ${ }^{2}$ Neurological Surgery and ${ }^{3}$ Neurology, \\ Northwestern University, Feinberg School of Medicine and McGaw Medical Center, Chicago, Illinois
}

\begin{abstract}
OBJECTIVE Intraoperative stimulation has emerged as a crucial adjunct in neurosurgical oncology, aiding maximal tumor resection while preserving sensorimotor and language function. Despite increasing use in clinical practice of this stimulation, there are limited data on both intraoperative seizure (IS) frequency and the presence of afterdischarges (ADs) in patients undergoing such procedures. The objective of this study was to determine risk factors for IS or ADs, and to determine the clinical consequences of these intraoperative events.

METHODS A retrospective chart review was performed for patients undergoing awake craniotomy (both first time and repeat) at a single institution from 2013 to 2018. Hypothesized risk factors for ADs/ISs in patients were evaluated for their effect on ADs and ISs, including tumor location, tumor grade (I-IV), genetic markers (isocitrate dehydrogenase $1 / 2,0^{6}$ methylguanine-DNA methyltransferase [MGMT] promoter methylation, chromosome 1p/19q codeletion), tumor volume, preoperative seizure status (yes/no), and dosage of preoperative antiepileptic drugs for each patient. Clinical outcomes assessed in patients with IS or ADs were duration of surgery, length of stay, presence of perioperative deficits, and postoperative seizures. Chi-square analysis was performed for binary categorical variables, and a Student t-test was used to assess continuous variables.
\end{abstract}

RESULTS A total of 229 consecutive patients were included in the analysis. Thirty-five patients (15\%) experienced ISs. Thirteen (37\%) of these 35 patients had experienced seizures that were appreciated clinically and noted on electrocorticography simultaneously, while 8 patients (23\%) experienced ISs that were electrographic alone (no obvious clinical change). MGMT promoter methylation was associated with an increased prevalence of ISs (OR 3.3, 95\% Cl 1.2-7.8, $\mathrm{p}$ $=0.02)$. Forty patients $(18 \%)$ experienced ADs. Twenty-three percent of patients $(9 / 40)$ with ISs had ADs prior to their seizure, although ISs and ADs were not statistically associated $(p=0.16)$. The presence of ADs appeared to be correlated with a shorter length of stay ( $5.1 \pm 2.6$ vs $6.1 \pm 3.7$ days, $p=0.037)$. Of the clinical features assessed, none were found to be predictive of ADs. Neither IS nor AD, or the presence of either IS or AD (65/229 patients), was a predictor for increased length of stay, presence of perioperative deficits, or postoperative seizures.

CONCLUSIONS ISs and ADs, while commonly observed during intraoperative stimulation for brain mapping, do not negatively affect patient outcomes.

https://thejns.org/doi/abs/10.3171/2020.3.JNS193231

KEYWORDS afterdischarges; electrocorticography; intraoperative stimulation; seizures; epilepsy

I NTRAOPERATIVE stimulation has emerged as a crucial adjunct in neurosurgical oncology, aiding maximal tumor resection while preserving sensorimotor and language function, ${ }^{1-3}$ and awake craniotomy has been shown to improve survival in high-grade gliomas. ${ }^{2}$ Despite its increasing use in clinical practice, there are limited data on intraoperative seizure (IS) frequency in patients undergo- ing cortical stimulation during awake craniotomy. Additionally, little is known about the clinical consequences of afterdischarges (ADs), defined as repetitive epileptiform discharges provoked by a stimulus ${ }^{4}$ in particular the potential for inaccurate functional mapping during surgery. Additionally, while the presence of postoperative seizures can be a significant burden to the healthcare system due to

ABBREVIATIONS $A D$ = afterdischarge; $A E D$ = antiepileptic drug; $E C O G$ = electrocorticography; $I D H=$ isocitrate dehydrogenase; $I S$ = intraoperative seizure; $M G M T$ = $0^{6}$-methylguanine-DNA methyltransferase; $\mathrm{OR}=$ odds ratio.

SUBMITTED November 29, 2019. ACCEPTED March 30, 2020.

INCLUDE WHEN CITING Published online May 22, 2020; DOI: 10.3171/2020.3.JNS193231. 
longer hospital stays and neurological morbidity, ${ }^{5}$ it is unclear whether or not there is a causal relationship between ADs and/or IS and postoperative seizures, and whether the underlying mechanisms are similar or different.

The objective of this study was to investigate whether known risk factors for preoperative or postoperative seizures were predictive for AD or IS. Thus, we evaluated the relationship between tumor grade, ${ }^{6-8}$ tumor location, ${ }^{9-11}$ tumor volume, ${ }^{8}$ tumor genetics $\left(O^{6}\right.$-methylguanine-DNA methyltransferase [MGMT] promoter methylation, isocitrate dehydrogenase $[I D H] 1 / 2$ mutation, chromosome $1 \mathrm{p} / 19 \mathrm{q}$ codeletion), ${ }^{6,12,13}$ history of seizures,${ }^{14}$ and the presence of either intraoperative ADs or IS. Additionally, we sought to better understand the clinical consequences, both intraoperatively and postoperatively, of patients experiencing AD or IS. Specifically, we asked whether the presence of ADs or IS led to 1) a significant increase in operative time, 2) new neurological deficits, 3) increased postoperative seizure activity, or 4) increased length of hospital stay.

\section{Methods \\ Cortical Mapping Protocol}

IRB approval was obtained to retrospectively review the charts of 229 patients undergoing awake craniotomy at our institution (Northwestern University) from 2013 through 2018. Awake craniotomy was offered to select patients with lesions near or within eloquent brain areas, including language, motor, and sensory areas. For awake surgery, prophylactic levetiracetam (500-1000 mg) was given prior to surgery, in addition to patients' prescribed antiepileptic drugs (AEDs) prior to admission. Intraoperative anesthesia included remifentanil and propofol, which was discontinued during the period of cortical mapping. Prior to tumor resection, positive cortical mapping was performed as previously described ${ }^{15}$ using a bipolar electrode with 5-mm spacing ( $60 \mathrm{~Hz}, 1-\mathrm{msec}$ pulse width, 1-6 $\mathrm{mA})$. The current intensity was started at $1 \mathrm{~mA}$ and increased by $0.5 \mathrm{~mA}$ until a positive mapping site (sensory, motor, speech, or language) was elicited. The lowest current capable of provoking a consistent response was used for subsequent cortical/subcortical mapping throughout the resection. At our institution, we use different stimulation methods for asleep motor mapping cases, specifically high-frequency stimulation. Based on prior data ${ }^{16}$ and on our institutional experience, we rarely observe IS/ADs in our asleep mapping population and thus would not have enough events for a reasonable comparison.

Integra grids of variable sizes including $1 \times 4(\mathrm{n}=159)$, $1 \times 6(\mathrm{n}=15), 4 \times 5(\mathrm{n}=16), 4 \times 8(\mathrm{n}=12)$, and $8 \times 8(\mathrm{n}$ $=2$ ) were used to monitor electrocorticography $(\mathrm{ECoG})$ during intraoperative mapping.

\section{Intraoperative Monitoring}

Per institutional protocol, ECoG was monitored for the entire period of cortical mapping. ECoG was recorded using either a Cadwell or Blackrock System (sampling frequency $=2000 \mathrm{~Hz}$, high-frequency filters $=300 \mathrm{~Hz}$, lowfrequency filters $=0.53 \mathrm{~Hz}$, sensitivity $=30-150 \mu \mathrm{V}$ ). An epileptologist and neuropsychologist were present in the operating room for all cases to provide real-time feedback to the neurosurgeon regarding ECoG interpretation, a patient's capacity to continue mapping after an IS/AD, and management of intraoperative ADs/ISs. The intraoperative neuromonitoring report was generated by the epileptologist for each awake craniotomy, which reported both ADs and ISs, if present. For each surgery, cortical stimulation intensity $(\mathrm{mA})$, duration of $\mathrm{AD} / \mathrm{IS}$, and total number of discrete $\mathrm{AD} / \mathrm{IS}$ events were noted.

\section{ECoG Review}

Of the 229 patients with ECoG recordings available for review, $19(8.3 \%)$ had ADs or ISs. ECoG recordings were reviewed by a board-certified epileptologist to calculate the number and duration of ADs and/or ISs. ADs were defined as repetitive epileptiform discharges that follow a preceding stimulus. ${ }^{4}$ ISs were defined as an evolution of ADs or as discharges that demonstrated an evolution in morphology or field, regardless of the duration. The number and duration of direct electrical stimulations were quantified for each patient using the signature ECoG artifact observed with cortical stimulation (Fig. 1).

\section{Retrospective Chart Review}

Using the electronic medical record, each patient's chart was reviewed to collect demographics, including the patient's age, sex, history of prior craniotomy, history of prior awake craniotomy, handedness, location of tumor, and histology (grade I-IV, MGMT promoter methylation, IDH $1 / 2$ mutation, and chromosome $1 \mathrm{p} / 19 \mathrm{q}$ codeletion). To better ascertain a patient's seizure or epilepsy state, two characteristics were collected from the chart. First, we identified if a seizure was the presenting symptom for consultation for resection (seizure history $=$ yes/no). Second, we identified the dosage of preoperative AEDs for each patient (two groups, i.e., patients receiving no AEDs at the time of surgery or the lowest possible dose for an AED vs those patients who had been uptitrated on their AEDS) to better understand the patient's preoperative seizure control. Procedure reports were used to identify the maximum current used by the surgeon for cortical stimulation, along with procedure duration. The electronic medical record was used to evaluate for any postoperative neurological deficits, and whether any postoperative clinical seizures were observed by the treating team. Patients with unexplained focal deficits, altered mental status out of proportion to that expected postoperatively, or overt seizure activity were monitored with continuous ECoG. ECoG reports were reviewed in conjunction with surgical procedure reports for documentation of seizures. Finally, the discharge summary was used to assess the length of stay for each patient.

\section{Radiological Findings}

For patients undergoing a first-time craniotomy, the preoperative tumor and tumor edema volumes were evaluated using T1-weighted postcontrast and FLAIR MRI sequences. The patient's preoperative MR images were uploaded into the Brainlab software (Brainlab Elements, version 4.0) and the tumor was manually traced using 


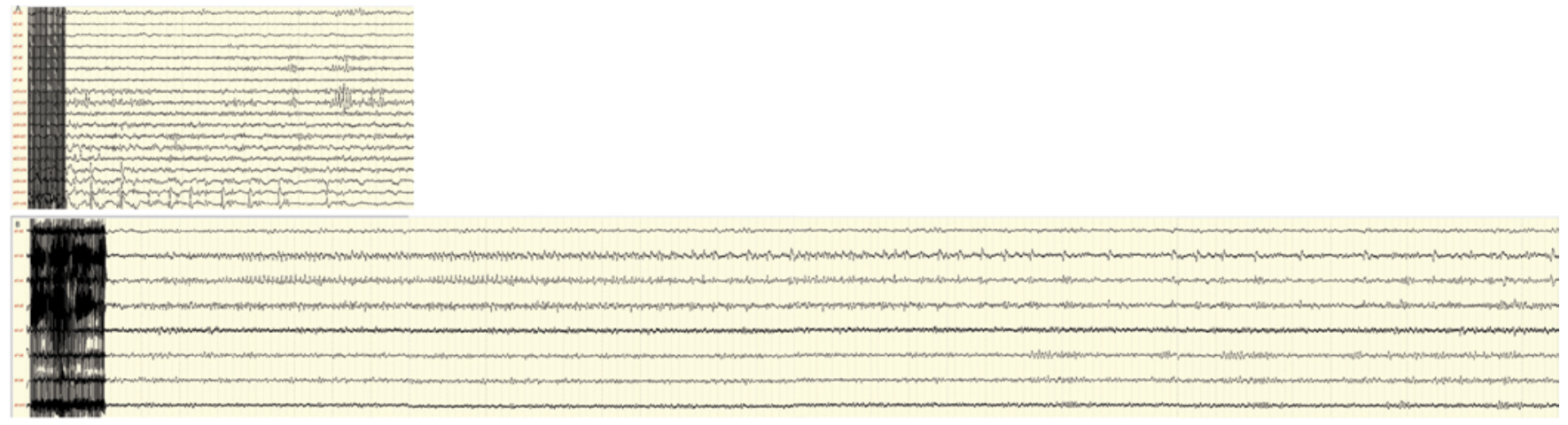

FIG. 1. Examples of both an IS and ADs captured with intraoperative ECoG. A: ADs noted as repetitive spikes that decrease in frequency over the course of 8 seconds on electrode a31. B: Electrographic IS noted at electrode a3 after stimulation lasting approximately 40 seconds. Figure is available in color online only.

the paintbrush feature in the sagittal, coronal, and axial planes. A 3D object was then generated, and the volume of the object was recorded. Patients undergoing repeat craniotomy for recurrent tumor were not included in this section of analysis.

\section{Statistical Analysis}

Statistical analysis was conducted using Prism 6 (GraphPad). Chi-square analysis (Baptista-Pike for odds ratio [OR]) was performed for binary categorical variables. For any continuous variables, a Student t-test was performed. A p value $<0.05$ was considered significant.

\section{Results}

\section{Intraoperative Seizures}

A total of 229 patients were included in our analysis. Based on the intraoperative neuromonitoring reports, a total of 35 patients (15\%) experienced IS. Fourteen (40\%) of these 35 patients with IS had clinical seizures without an electrographic correlate. Importantly, 8 ISs (23\%) were electrographic alone (no obvious clinical change), while $13(37 \%)$ were appreciated clinically and noted on ECoG simultaneously. Of the 27 patients with clinical seizures, $23(85 \%)$ had focal seizures and $4(15 \%)$ had generalized seizures. Twenty patients had ISs that resolved spontaneously, 12 had ISs that abated with use of cold saline, and 5 of the patients receiving cold saline also required additional AED administration, whereas 3 patients received only additional intraoperative AEDs. Only 2 awake procedures were converted to asleep surgeries as a direct result of seizures (2/35 ISs, 2/229 patients within the series).

Of the patients who had ISs as reported by ECoG recording (5/35), they experienced a mean of $2.2 \pm 1.6$ seizures, each lasting $114 \pm 54$ seconds. The average number of cortical stimulations by the surgeon was $60 \pm 45$, with each stimulation lasting $2.19 \pm 0.71$ seconds.

\section{Intraoperative $A D$}

Forty patients (17\%) had ADs during intraoperative mapping. In 9 patients (3.9\%), both ADs and ISs were observed. For 15 patients with ADs, a detailed ECoG review revealed that patients had 2.3 discrete episodes of ADs, with each lasting $8.7 \pm 5.6$ seconds. The average number of cortical stimulations by the surgeon was $72 \pm 48$, with each stimulation lasting on average $2.2 \pm 0.6$ seconds. ADs were noted to occur prior to ISs in 9 (23\%) of the 40 cases.

\section{Risk Factors for Intraoperative AD/IS}

$M G M T$ methylation was associated with an increased frequency of ISs (OR 3.04, 95\% CI 1.2-7.8, $\mathrm{p}=0.021$; Table 1) but not for intraoperative ADs (OR 0.99, 95\% CI $0.46-2.1, \mathrm{p}=0.97$; Table 2). Tumor location, IDH mutational status, preoperative seizure control, and intensity of cortical stimulation were not predictive of either IS or AD. As part of the institutional intraoperative protocol, patients received a dose of levetiracetam (500-1000 $\mathrm{mg}$ ) prior to surgery. One hundred thirty-one patients were receiving either no AED (78/229, 34\%) or the minimum dosage of a single AED $(53 / 229,23 \%)$, whereas 91 patients $(91 / 229$, $40 \%$ ) had been uptitrated either on an AED $(65 / 91,71 \%)$ or on multiple agents $(26 / 91,29 \%)$. Interestingly, both patients who presented with preoperative seizures (IS OR $0.88,95 \%$ CI $0.41-1.8, \mathrm{p}=0.73$; AD OR $0.70,95 \%$ CI $0.35-1.4, \mathrm{p}=0.30)$ and AEDs greater than the starting dose (IS OR 1.4, 95\% CI 0.69-2.8, p = 0.36; AD OR 0.65, $95 \%$ CI $0.33-1.3, \mathrm{p}=0.25$ ) did not predict IS or AD, nor were they predictive of the presence of postoperative seizures (OR 0.66, 95\% CI 0.31-1.4, $\mathrm{p}=0.27$ ).

\section{Perioperative Morbidity}

Neither IS (Table 1) nor ADs (Table 2) had a significant effect on operative duration (IS mean $236 \pm 56.5$ vs $230 \pm$ 65.7 minutes, $\mathrm{p}=0.56$; AD $237 \pm 38$ vs $229 \pm 69$ minutes, $\mathrm{p}=0.34$; both IS and AD $236 \pm 38$ vs $230 \pm 65$ minutes, $p$ $=0.46$; Table 3). Neither IS nor ADs statistically increased the chance of postoperative seizures (IS OR 1.3, 95\% CI $0.51-3.3, \mathrm{p}=0.57$; AD OR $0.51,95 \%$ CI $0.19-1.5, \mathrm{p}=0.22$; both IS and AD OR 0.97, 95\% CI 0.20-4.6, p = 0.97), a new or worsened neurological deficit in the perioperative period (IS OR $0.50,95 \%$ CI $0.23-1.1, \mathrm{p}=0.10 ; \mathrm{AD}$ OR 1.06, 95\% CI 0.50-2.2, p = 0.88; both IS and AD OR $0.55,95 \%$ CI $0.11-2.5, \mathrm{p}=0.44$ ), or a postoperative complication such as an infection or a wound dehiscence (IS OR 1.3, 95\% CI 0.4-4.0, $\mathrm{p}=0.67$; AD OR 1.47, 95\% CI $0.41-5.2, \mathrm{p}=0.58$; both IS and AD OR 0, 95\% CI 0-4.4, 
TABLE 1. Summary of IS patient characteristics $(n=35)$

\begin{tabular}{|c|c|c|c|c|c|}
\hline Variable & IS & No IS & OR & $95 \% \mathrm{Cl}$ & $p$ Value \\
\hline Males & 20 & 105 & 1.13 & $0.50-2.1$ & 0.74 \\
\hline Females & 15 & 89 & 0.95 & $0.47-1.99$ & 0.90 \\
\hline Mean age $\pm S D$, yrs & $50.2 \pm 12.9$ & $48.6 \pm 17.1$ & & & 0.53 \\
\hline Prior resection & 16 & 64 & 1.60 & $0.78-3.38$ & 0.20 \\
\hline Repeat awake craniotomy & 3 & 11 & 1.50 & $0.43-5.4$ & 0.55 \\
\hline History of seizure & 20 & 117 & 0.88 & $0.41-1.8$ & 0.73 \\
\hline AED > starting dose & 17 & 76 & 1.4 & $0.69-2.8$ & 0.36 \\
\hline \multicolumn{6}{|l|}{ Tumor location } \\
\hline $\mathrm{Rt}$ & 12 & 64 & 0.99 & $0.47-2.1$ & 0.98 \\
\hline $\mathrm{Lt}$ & 23 & 128 & 1.01 & $0.47-2.15$ & 0.98 \\
\hline Frontal & 18 & 114 & 0.693 & $0.35-1.4$ & 0.31 \\
\hline Parietal & 17 & 64 & 1.9 & $0.94-3.9$ & 0.076 \\
\hline Temporal & 9 & 46 & 1.1 & $0.45-2.3$ & 0.88 \\
\hline Occipital & 2 & 13 & - & - & - \\
\hline Insular & 1 & 7 & - & - & - \\
\hline Other & 2 & 5 & - & - & - \\
\hline \multicolumn{6}{|l|}{ Tumor histology } \\
\hline Grade I & 1 & 10 & - & - & - \\
\hline Grade II & 4 & 28 & - & - & - \\
\hline Grade III & 11 & 42 & 1.8 & $0.85-4.2$ & 0.14 \\
\hline Grade IV & 14 & 95 & 0.74 & $0.35-1.6$ & 0.44 \\
\hline Grade I-II & 5 & 38 & 0.72 & $0.28-1.9$ & 0.53 \\
\hline Grade III-IV & 25 & 137 & 1.4 & $0.52-3.5$ & 0.53 \\
\hline MGMT methylation & 19 & 72 & 3.04 & $1.2-7.8$ & $0.021^{*}$ \\
\hline IDH 1/2 mutation & 13 & 73 & 1.0 & $0.47-2.3$ & 0.95 \\
\hline Periop deficits & 7 & 66 & 0.50 & $0.23-1.1$ & 0.10 \\
\hline Periop seizures & 7 & 31 & 1.3 & $0.51-3.3$ & 0.57 \\
\hline Complications & 3 & 32 & 1.3 & $0.4-4.0$ & 0.67 \\
\hline Mean current amplitude $\pm \mathrm{SD}, \mathrm{mA}$ & $2.1 \pm 0.84$ & $2.1 \pm 0.81$ & - & - & 0.99 \\
\hline Mean length of surgery $\pm \mathrm{SD}$, mins & $236 \pm 56.5$ & $230 \pm 65.7$ & - & - & 0.56 \\
\hline Mean length of stay $\pm S D$, days & $5.3 \pm 2.6$ & $6.1 \pm 3.6$ & - & - & 0.15 \\
\hline Mean FLAIR tumor volume \pm SD $\dagger$ & $96 \pm 147$ & $73 \pm 63$ & - & - & 0.63 \\
\hline Mean $\mathrm{T} 1+\mathrm{C}$ tumor volume $\pm \mathrm{SD} \dagger$ & $28 \pm 20$ & $37 \pm 36$ & - & - & 0.34 \\
\hline
\end{tabular}

$\mathrm{p}=0.40$ ). Patients who had ADs intraoperatively had a shorter length of stay as compared to those without (5.1 \pm 2.6 vs $6.2 \pm 3.7$ days, $p=0.037$ ), whereas there was no difference seen in the other analyzed groups (IS $5.3 \pm 2.6$ vs $6.1 \pm 3.6$ days, $\mathrm{p}=0.15$; both IS and AD $6.0 \pm 3.5$ vs $6.0 \pm 3.5$ days, $p=0.99$ ). Further analysis was conducted to evaluate postoperative outcomes in the patient group in which an IS was observed. Based on the intraoperative neuromonitoring report and the procedure report generated by the surgeon, the patients were categorized as having a clinical seizure (witnessed seizure activity) or subclinical seizure (no overt seizure was witnessed, but ECoG reading was indicative of a seizure) intraoperatively. No difference was appreciated in any postoperative outcome (ECoG vs clinical; surgery duration $223 \pm 50.37$ vs 240 \pm 59.4 minutes, $\mathrm{p}=0.47$; postoperative length of stay 4.1 \pm 1.7 vs $5.8 \pm 2.7$ days, $p=0.07$ ). Furthermore, patients with a clinical seizure did not have a significantly different incidence of new postoperative deficits as compared to the subclinical seizure group (OR 0.5, 95\% CI 0.04-3.7, $\mathrm{p}=0.54$ ). Patients with a clinical seizure did not have an increased incidence of postoperative seizures as compared to the rest of the patients (OR 1.5, 95\% CI 0.62-2.7, $\mathrm{p}=$ $0.33)$. Within the group of patients who had intraoperative clinical seizures, patients with a generalized seizure did not have a significantly different surgical duration or length of stay as compared to those with a focal seizure (duration of surgery $236 \pm 103$ vs $232 \pm 41$ minutes, $\mathrm{p}=$ 0.96 ; length of stay $8.0 \pm 3.6$ vs $5.3 \pm 2.4$ days, $\mathrm{p}=0.22$ ). Patients with a generalized seizure also did not have a sig- 
TABLE 2. Summary of patients with ADs $(n=40)$

\begin{tabular}{|c|c|c|c|c|c|}
\hline Variable & Intraop AD & No Intraop AD & OR & $95 \% \mathrm{Cl}$ & $p$ Value \\
\hline Males & 23 & 102 & 1.15 & $0.59-2.24$ & 0.68 \\
\hline Females & 17 & 87 & 0.87 & $0.45-1.7$ & 0.68 \\
\hline Mean age $\pm S D$, yrs & $51 \pm 23.2$ & $48 \pm 14.8$ & & & 0.56 \\
\hline Prior resection & 8 & 72 & - & & \\
\hline Repeat awake craniotomy & 1 & 13 & 0.35 & $0.032-1.97$ & 0.29 \\
\hline History of seizure & 21 & 116 & 0.70 & $0.35-1.4$ & 0.30 \\
\hline AED > starting dose & 13 & 80 & 0.65 & $0.33-1.3$ & 0.25 \\
\hline \multicolumn{6}{|l|}{ Tumor location } \\
\hline $\mathrm{Rt}$ & 9 & 67 & 0.52 & $0.23-1.2$ & 0.11 \\
\hline $\mathrm{Lt}$ & 31 & 120 & 1.92 & $0.87-4.4$ & 0.11 \\
\hline Frontal & 28 & 104 & 1.91 & $0.93-3.92$ & 0.082 \\
\hline Parietal & 12 & 69 & 0.75 & $0.36-1.50$ & 0.43 \\
\hline Temporal & 10 & 45 & 1.07 & $0.50-2.4$ & 0.87 \\
\hline Occipital & 1 & 14 & - & - & - \\
\hline Insular & 1 & 7 & - & - & - \\
\hline Other & 1 & 6 & - & - & - \\
\hline \multicolumn{6}{|l|}{ Tumor histology } \\
\hline Grade I & 1 & 10 & - & - & - \\
\hline Grade II & 7 & 25 & - & - & - \\
\hline Grade III & 13 & 40 & 1.58 & $0.77-3.4$ & 0.24 \\
\hline Grade IV & 18 & 91 & 0.71 & $0.36-1.4$ & 0.33 \\
\hline Grade I-II & 8 & 35 & 0.97 & $0.43-2.3$ & 0.94 \\
\hline Grade III-IV & 31 & 131 & 1.04 & $0.44-2.34$ & 0.94 \\
\hline MGMT methylation & 18 & 73 & 0.99 & $0.46-2.1$ & 0.97 \\
\hline IDH $1 / 2$ mutation & 18 & 68 & 1.17 & $0.59-2.6$ & 0.67 \\
\hline Periop deficits & 14 & 59 & 1.06 & $0.50-2.2$ & 0.88 \\
\hline Periop seizures & 4 & 36 & 0.51 & $0.19-1.5$ & 0.22 \\
\hline Complications & 3 & 37 & 1.47 & $0.41-5.2$ & 0.58 \\
\hline Mean current amplitude $\pm \mathrm{SD}$, mA & $2.3 \pm 0.73$ & $2.1 \pm 0.82$ & - & - & 0.10 \\
\hline Mean length of surgery $\pm S D$, mins & $237 \pm 38$ & $229 \pm 69$ & - & - & 0.34 \\
\hline Mean length of stay $\pm S D$, days & $5.1 \pm 2.6$ & $6.2 \pm 3.7$ & - & - & $0.040^{*}$ \\
\hline Mean FLAIR tumor volume \pm SD $†$ & $109 \pm 114$ & $65 \pm 58$ & - & - & 0.074 \\
\hline Mean T1+C tumor volume \pm SD $†$ & $33 \pm 27$ & $38 \pm 37$ & - & - & 0.62 \\
\hline
\end{tabular}

* Significant value $(p<0.05)$.

$\dagger$ First-time resection.

nificantly different incidence of postoperative deficits (OR $1.2,95 \%$ CI $0.08-9.6, \mathrm{p}=0.88$ ) or postoperative seizures (OR $0.94,95 \%$ CI $0.06-7.5, \mathrm{p}=0.96$ ) as compared to those with focal seizures.

There were 65 patients (28\%) who had either an intraoperative AD or IS. We evaluated whether these patients were at a higher risk for perioperative morbidity. No difference was appreciated in patients with either an IS or intraoperative $\mathrm{AD}$ as compared to patients without $\mathrm{AD} / \mathrm{IS}$ (duration of surgery $237 \pm 47.9$ vs $228 \pm 69.5$ minutes, $p$ $=0.30$; postoperative seizures OR $0.86,95 \%$ CI $0.4-1.9, \mathrm{p}$ $=0.71$; postoperative deficits OR $0.82,95 \%$ CI $0.45-1.5, \mathrm{p}$ $=0.52$ ). However, patients without IS or AD had a longer length of stay as compared to patients who experienced them $(6.3 \pm 3.8$ vs $5.1 \pm 2.4$ days, $\mathrm{p}=0.007)$.

\section{Discussion}

This study investigates the incidence, risk factors, and clinical impact of intraoperative ADs and ISs in a large awake craniotomy cohort. Most significantly, we found no major differences in operative duration, postoperative seizures, new neurological deficits, or length of hospital stay for patients experiencing intraoperative ADs/ISs. Our reported incidence for ISs was $15.2 \%$, which is consistent with reports from previous studies ranging from $8.8 \%$ to $18.6 \% .{ }^{17-19}$ Additionally, IS or AD activity during awake craniotomy rarely led to a significant change in operative plan and did not significantly lengthen the duration of surgery. There did not appear to be a clear relationship between intraoperative ADs and progression to IS, and less than $5 \%$ of patients had both intraoperative ADs and 
TABLE 3. Summary of patients in whom both ISs and ADs were present in the same case $(n=9)$

\begin{tabular}{|c|c|c|c|c|c|}
\hline Variable & ISs + ADs & Other & OR & $95 \% \mathrm{Cl}$ & $p$ Value \\
\hline Males & 5 & 120 & 1.0 & $0.30-3.5$ & 0.95 \\
\hline Females & 4 & 100 & 0.96 & $0.29-3.3$ & 0.95 \\
\hline Mean age $\pm S D$, yrs & $49.8 \pm 14.5$ & $48.8 \pm 16.6$ & & & 0.85 \\
\hline Prior resection & 4 & 76 & 1.5 & $0.45-5.2$ & 0.55 \\
\hline Repeat awake craniotomy & 1 & 13 & 2.0 & $0.17-12$ & 0.52 \\
\hline History of seizure & 4 & 133 & 0.50 & $0.16-1.8$ & 0.34 \\
\hline \multicolumn{6}{|l|}{ Tumor location } \\
\hline $\mathrm{Rt}$ & 2 & 74 & 0.60 & $0.11-2.6$ & 0.47 \\
\hline $\mathrm{Lt}$ & 7 & 144 & 1.8 & $0.38-8.7$ & 0.47 \\
\hline Frontal & 5 & 114 & 0.70 & $0.35-1.4$ & 0.31 \\
\hline Parietal & 4 & 77 & 1.50 & $0.44-5.1$ & 0.56 \\
\hline Temporal & 1 & 54 & 0.38 & $0.034-2.6$ & 0.36 \\
\hline \multicolumn{6}{|l|}{ Tumor histology } \\
\hline Grade III & 1 & 52 & 0.35 & $0.030-2.4$ & 0.30 \\
\hline Grade IV & 5 & 104 & 1.1 & $0.32-3.7$ & 0.88 \\
\hline Grade I-II & 3 & 40 & 2.0 & $0.51-7.3$ & 0.35 \\
\hline Grade III-IV & 6 & 156 & 0.51 & $0.14-1.9$ & 0.35 \\
\hline MGMT methylation & 5 & 86 & 2.1 & $0.43-10.9$ & 0.37 \\
\hline IDH $1 / 2$ mutation & 4 & 82 & 1.0 & $0.30-3.5$ & 0.99 \\
\hline Periop deficits & 2 & 71 & 0.55 & $0.11-2.5$ & 0.44 \\
\hline Periop seizures & 2 & 7 & 0.97 & $0.2-4.6$ & 0.97 \\
\hline Complications & 0 & 9 & 0 & $0-4.4$ & 0.40 \\
\hline Mean current amplitude $\pm \mathrm{SD}, \mathrm{mA}$ & $2.1 \pm 0.7$ & $2.1 \pm 0.8$ & - & - & 0.81 \\
\hline Mean length of surgery $\pm S D$, mins & $236 \pm 38$ & $230 \pm 65$ & - & - & 0.46 \\
\hline Mean length of stay $\pm S D$, days & $6.0 \pm 3.5$ & $6.0 \pm 3.5$ & - & - & 0.99 \\
\hline Mean FLAIR tumor volume \pm SD $†$ & $173 \pm 199$ & $70 \pm 61$ & - & - & 0.35 \\
\hline Mean $\mathrm{T} 1+\mathrm{C}$ tumor volume $\pm \mathrm{SD} \dagger$ & $35 \pm 17$ & $36 \pm 35$ & - & - & 0.91 \\
\hline
\end{tabular}

† First-time resection.

ISs. Finally, approximately $25 \%$ of ISs were electrographic alone without any clinical signs of overt seizure activity. This finding reinforces the necessity for ECoG use in the operating room to better evaluate subclinical seizure activity, as interpreting mapping results in a patient experiencing IS may not be accurate and lead to false-positive or false-negative findings.

In the present study, tumor $M G M T$ methylation was associated with an increased risk of IS. In contrast, there were no predictors for patients with ADs. Given that MGMT methylation emerged as a predictor of IS, one would expect that low-grade gliomas would also be predictive given that a higher percentage have MGMT methylation compared to high-grade gliomas. However, this was not the case in the present study, perhaps due to a disproportionate number of patients who had high- versus low-grade gliomas in our cohort (162 vs 43 patients, respectively). Alternatively, MGMT methylation status, independent of grade, may predispose to IS. It is surprising that several risk factors for clinical seizures in glioma patients did not predict IS or AD, ${ }^{6-11,13,14}$ suggesting that intraoperative AD or IS may have a different physiological basis relative to normal seizures, and/or AD or IS may represent artificial or nonphysiological charge propagation. The lack of a relationship between IS and postoperative seizures is also consistent with this notion of distinct seizure propagation mechanisms.

Other studies have identified a variety of factors that lead to an increase in IS, including the presence of preoperative seizures, ${ }^{19} I D H 1$ mutation, ${ }^{12}$ supplementary motor area location, ${ }^{12}$ younger age, history of seizure, frontal location, and lower-grade gliomas. ${ }^{18}$ In addition to patient factors, a small number of studies have investigated whether the surgical approach itself has any effect, i.e., specifically if scalp blocks and dexmedetomidine are associated with ISs in patients undergoing awake craniotomy, but did not find this to be a significant contributor. ${ }^{19,20}$ As evidenced from the variety of variables that have been found across centers, it is difficult to appreciate a reproducible risk factor that would aid in predicting IS. To date, no papers have been published discussing predictors of ADs.

A recent study including 544 patients undergoing intraoperative mapping for both awake and asleep craniotomies showed that administering AEDs prior to surgery could potentially decrease the risk of IS; $;^{21}$ these findings were supported in a large retrospective review of 1916 patients 
undergoing craniotomy. ${ }^{22}$ As all of our patients received prophylactic AEDs, we were not able to either support or refute these findings.

The most significant finding of our study is that patients with ADs or IS did not have any significant clinical differences in the postoperative period; specifically, there were no differences in perioperative morbidity or in length of stay. These findings are in contrast to Nossek et al.'s results, which highlighted a higher likelihood of transient motor deficits $(12 / 60 \text { patients, or } 20 \% \text { vs } 10.1 \%, \mathrm{p}=0.02)^{18}$ in their patients in addition to a 1-day-longer hospital stay (4 vs 3 days, $p=0.045$ ) for patients who had IS. Surprisingly, we found that patients with ADs tend to stay in the hospital about 1 day less than other patients $(5.1 \pm 2.6$ vs $6.1 \pm 3.7$ days, $p=0.037$ ). There is not a clear explanation for this finding, although it is possible that this can be attributed to the relatively small sample size of ADs in the study, especially given the standard deviations noted in both groups. To ensure this was not due to IS being included in the other group, we also evaluated patients that had either AD or IS $(n=65)$ and still found a significant difference $(6.3 \pm 3.8$ vs $5.1 \pm 2.4$ days, $p=0.007)$. Although some studies have begun to investigate the effects of IS, there remains a paucity of literature regarding the effect of IS on clinically useful outcomes such as length of stay or the presence of postoperative seizures.

These findings are particularly reassuring as ISs are often misconceived to be a predictor of increased perioperative morbidity. In fact, most surgeons cite IS as a potential risk for awake craniotomies. Furthermore, in settings in which mapping is conducted by increasing the applied stimulation current until ADs are present, such as negative mapping protocols,,$^{23}$ our findings reassure that the presence of ADs does not have deleterious long-term effects. Our finding of no difference in outcomes in the patient subgroups with intraoperative clinical versus subclinical seizures further reinforces this idea.

Although $23 \%$ of patients with ADs went on to have ISs, this was not statistically significant $(\mathrm{p}=0.1627)$, and only 4\% (9/229) of all patients mapped had both ADs and ISs. Given the nature of ADs, this is likely due to surgeons being diligent about ceasing stimulation when ADs are detected, again highlighting the importance of intraoperative ECoG during mapping.

In our series, there were only 2 patients $(0.87 \%)$ who were unable to complete cortical mapping due to seizures. The only other study where this information was documented was in the case series reported by Nossek et al., in which 11 patients $(2.3 \%)$ were unable to be mapped due to overt seizure activity. ${ }^{24}$

\section{Study Limitations}

There are a few key limitations in this study. First and foremost, our review is retrospective in nature, so there is a potential for bias. Additionally, our assessment for the presence and duration of ADs or ISs for some patients, especially from earlier years, was limited to an intraoperative neuromonitoring report within the patient's chart. Second, as our epileptologists adopt the practice of using larger grids for neuromonitoring, it is possible that more ADs and ISs were detected compared to other studies, when smaller grids were utilized more routinely. Given the heterogeneity of grid sizes, there is a possibility that subclinical seizure activity or ADs were not identified due to lack of coverage. Additionally, the definition of $\mathrm{AD}$ versus IS was at the discretion of the epileptologist, who used clinical discretion to delineate between a prolonged $\mathrm{AD}$ and a standalone phenomenon that would be defined as an IS. Although the typical limit to an AD is approximately 10 seconds, as noted by the recordings, there were instances in which longer ADs were documented. In regard to evaluating patients with postoperative seizures, patients were only evaluated with ECoG if there was clinical concern for seizure activity. Therefore, it is possible that seizures went unnoticed. Finally, given the relatively low number of both $\mathrm{AD}$ and IS events, the overall power of the study is relatively low, particularly in the context of multiple comparisons. Also, these data are relevant to Ojemann/Penfield type stimulation, but may not pertain to other stimulation paradigms, such as high frequency. ${ }^{16}$

\section{Conclusions}

In this retrospective, single-center study, we reviewed the outcomes and patient characteristics for 229 patients undergoing awake craniotomy. We determined that $M G M T$ methylation increased the risk for an IS. We did not find any meaningful relationship between IS or AD as predictors for postoperative seizure activity, increased length of stay, or the presence of postoperative deficits. As ISs are a known risk for intraoperative mapping, it is reassuring to neurosurgeons that neither ADs nor ISs have a significant impact on either perioperative seizure activity, presence of new neurological deficits, or length of stay, and less than $1 \%$ of awake mapping sessions were halted due to seizure activity. These data reinforce the use of both positive and negative mapping to ensure maximum extent of resection.

\section{References}

1. De Witt Hamer PC, Robles SG, Zwinderman AH, et al. Impact of intraoperative stimulation brain mapping on glioma surgery outcome: a meta-analysis. J Clin Oncol. 2012;30(20):2559-2565.

2. Gerritsen JKW, Arends L, Klimek M, et al. Impact of intraoperative stimulation mapping on high-grade glioma surgery outcome: a meta-analysis. Acta Neurochir (Wien). 2019;161(1):99-107.

3. Joswig H, Bratelj D, Brunner T, et al. Awake craniotomy: first-year experiences and patient perception. World Neurosurg. 2016;90:588-596.e2.

4. Blume WT, Jones DC, Pathak P. Properties of after-discharges from cortical electrical stimulation in focal epilepsies. Clin Neurophysiol. 2004;115(4):982-989.

5. Dewan MC, White-Dzuro GA, Brinson PR, et al. Perioperative seizure in patients with glioma is associated with longer hospitalization, higher readmission, and decreased overall survival. J Neurosurg. 2016;125(4):1033-1041.

6. Feyissa AM, Worrell GA, Tatum WO, et al. Potential influence of IDH1 mutation and MGMT gene promoter methylation on glioma-related preoperative seizures and postoperative seizure control. Seizure. 2019;69:283-289.

7. Mezue WC, Ndubuisi CA, Chikani MC, et al. Epilepsy in primary intracranial tumors in a neurosurgical hospital in Enugu, South-East Nigeria. Niger J Clin Pract. 2015;18(5):681686. 
8. Skardelly M, Brendle E, Noell S, et al. Predictors of preoperative and early postoperative seizures in patients with intra-axial primary and metastatic brain tumors: A retrospective observational single center study. Ann Neurol. 2015;78(6):917-928.

9. Wu A, Weingart JD, Gallia GL, et al. Risk factors for preoperative seizures and loss of seizure control in patients undergoing surgery for metastatic brain tumors. World Neurosurg. 2017;104:120-128.

10. Yang P, You G, Zhang W, et al. Correlation of preoperative seizures with clinicopathological factors and prognosis in anaplastic gliomas: a report of 198 patients from China. Seizure. 2014;23(10):844-851.

11. You G, Sha ZY, Yan W, et al. Seizure characteristics and outcomes in 508 Chinese adult patients undergoing primary resection of low-grade gliomas: a clinicopathological study. Neuro Oncol. 2012;14(2):230-241.

12. Gonen T, Grossman R, Sitt R, et al. Tumor location and IDH1 mutation may predict intraoperative seizures during awake craniotomy. J Neurosurg. 2014;121(5):1133-1138.

13. Yang $\mathrm{P}$, Liang $\mathrm{T}$, Zhang $\mathrm{C}$, et al. Clinicopathological factors predictive of postoperative seizures in patients with gliomas. Seizure. 2016;35:93-99.

14. Kahlenberg CA, Fadul CE, Roberts DW, et al. Seizure prognosis of patients with low-grade tumors. Seizure. 2012;21(7):540-545.

15. Boetto J, Bertram L, Moulinié G, et al. Low rate of intraoperative seizures during awake craniotomy in a prospective cohort with 374 supratentorial brain lesions: electrocorticography is not mandatory. World Neurosurg. 2015;84(6):18381844.

16. Tate MC, Guo L, McEvoy J, Chang EF. Safety and efficacy of motor mapping utilizing short pulse train direct cortical stimulation. Stereotact Funct Neurosurg. 2013;91(6):379-385.

17. Eseonu CI, Rincon-Torroella J, Lee YM, et al. Intraoperative seizures in awake craniotomy for perirolandic glioma resections that undergo cortical mapping. J Neurol Surg A Cent Eur Neurosurg. 2018;79(3):239-246.

18. Nossek E, Matot I, Shahar T, et al. Intraoperative seizures during awake craniotomy: incidence and consequences: analysis of 477 patients. Neurosurgery. 2013;73(1):135-140.

19. Spena G, Schucht P, Seidel K, et al. Brain tumors in eloquent areas: a European multicenter survey of intraoperative mapping techniques, intraoperative seizures occurrence, and antiepileptic drug prophylaxis. Neurosurg Rev. 2017;40(2):287298.
20. McAuliffe N, Nicholson S, Rigamonti A, et al. Awake craniotomy using dexmedetomidine and scalp blocks: a retrospective cohort study. Can J Anaesth. 2018;65(10):1129-1137.

21. Dineen J, Maus DC, Muzyka I, et al. Factors that modify the risk of intraoperative seizures triggered by electrical stimulation during supratentorial functional mapping. Clin Neurophysiol. 2019;130(6):1058-1065.

22. Kutteruf R, Yang JT, Hecker JG, et al. Incidence and risk factors for intraoperative seizures during elective craniotomy. $J$ Neurosurg Anesthesiol. 2019;31(2):234-240.

23. Sanai N, Mirzadeh Z, Berger MS. Functional outcome after language mapping for glioma resection. $N$ Engl J Med. 2008;358(1):18-27.

24. Nossek E, Matot I, Shahar T, et al. Failed awake craniotomy: a retrospective analysis in 424 patients undergoing craniotomy for brain tumor. J Neurosurg. 2013;118(2):243-249.

\section{Disclosures}

Dr. Ayer reports ownership in, being a patent holder for, and direct stock ownership in Rhaeos Inc. and Hubly Surgical Inc.

\section{Author Contributions}

Conception and design: Tate, Abecassis, Ayer, Templer. Acquisition of data: all authors. Analysis and interpretation of data: all authors. Drafting the article: Tate, Abecassis, Templer, Yerneni. Critically revising the article: all authors. Reviewed submitted version of manuscript: Tate, Abecassis, Ayer, Templer, Yerneni. Approved the final version of the manuscript on behalf of all authors: Tate. Statistical analysis: Abecassis, Yerneni. Administrative/technical/material support: Tate, Ayer, Templer. Study supervision: Tate, Ayer, Templer.

\section{Supplemental Information}

\section{Previous Presentations}

Portions of this work were presented in poster form at the CNS Annual Meeting in Houston, Texas, in October 2018.

\section{Correspondence}

Matthew C. Tate: Northwestern University, Feinberg School of Medicine and McGaw Medical Center, Chicago, IL. mtate@ nm.org. 